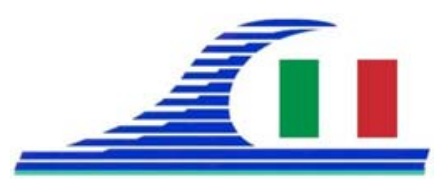

\author{
Conférence Méditerranéenne Côtière et Maritime \\ EDITION 3, FERRARA, ITALIA (2015) \\ Coastal and Maritime Mediterranean Conference \\ Disponible en ligne - http://www.paralia.fr - Available online
}

\title{
Exploitation des sables de plage et des dunes côtières. Impact sur l'équilibre du littoral entre Boumerdès et Zemmouri El Bahri. (Est-Algérois) Algérie
}

\author{
Hanifa HABIKI ${ }^{1}$, Lazreg BENSLAMA ${ }^{1}$
}

1. Laboratoire de Géo-Environnement, U. des Sciences et Technologie H. Boumédiène. Alger, Algérie.hbenslama@gmail.com

\section{Résumé}

La surexploitation, des sables des dunes littorales et des plages, occasionne un déséquilibre du littoral déjà fragile et expose ce dernier à l'action agressive de la mer. Le cas de la plage de Zemmouri el-bahri et Boumerdès est édifiant. La plage est bordée par un ensemble de deux dunes littorales juxtaposées. L'une longe directement la plage, elle est communément appelée dune vive, l'autre nommée dune ancienne occupe la deuxième position vers le continent. Deux phénomènes principaux, d'une part l'action érosive de la mer et d'autre part la surexploitation des sablières sont la cause majeure de la dégradation du littoral. On assiste à une disparition de la dune vive et une destruction partielle de la dune ancienne. Par endroit des dénivelés se créent et facilitent la remontée des eaux marines. Pour étudier ce phénomène, nous avons effectué des profils de plages avec une fréquence d’un levé tous les deux mois pendant une année.

Mots clés : Littoral, Plage, Zemmouri El Bahri, Boumerdès, Sablières, Dunes, Profils.

\section{Matériels et méthode}

Le nivellement a été réalisé à l'aide d'un théodolite T16 Wild et d'une mire parlante. L'appareil mis en station en tête de profil, il a été opéré à chaque inflexion (changement de pente visible) des mesures simultanées de distance par visée directe et d'altimétrie par visée horizontale sur la mire.

\section{Introduction}

Selon PASKOFF (2010) le niveau moyen des mers peut être perturbé par des oscillations limitées dans le temps, telles les vagues et les légères fluctuations dues à des phénomènes saisonniers (variation du débit des courants océaniques, de la pression atmosphérique, des vents dominants et de la densité de l'eau de mer). Les phénomènes majeurs pouvant façonner le littoral sont la marée et le déferlement des vagues. La marée est de très faible amplitude voire pratiquement nulle sur les côtes algériennes, d'où son influence insignifiante.

La baie de Zemmouri est très ouverte à l'influence des conditions océanologiques. De ce fait, on assiste à des variations, parfois spectaculaires, du trait de côte. 
Côtes méditerranéennes menacées :

Risques et défis dans le contexte du changement climatique

Les différentes plages aériennes sont bordées à leur extrémité sud par des dunes vives et des dunes anciennes plus ou moins fixées par la végétation. Ces dunes constituent une protection naturelle, d'où un équilibre dynamique de plage.

Les apports détritiques de l'oued Isser essentiellement et pour une moindre part ceux issus de l'oued Sébaou participent à l'alimentation des plages sous-marines et aériennes ainsi que les rides d'avant côte.

Le matériel grossier, supérieur à $500 \mu \mathrm{m}$, amené lors des crues se dépose aux embouchures puis est redistribué tangentiellement par les courants de dérives littorale. Il constitue dans la plupart des endroits le jet de rive dont une partie est reprise par les courants de retour (rip-current) alimentant ainsi les dunes hydrauliques par petits fonds.

Ces rides construites par les houles, introduisent deux types d'irrégularités dans la répartition granulométrique. Le sommet des rides est souligné par la présence de sédiments grossiers (médiane supérieure à $300 \mu \mathrm{m}$ ) du fait de l'agitation plus grande de l'eau sous l'effet des déferlements de la houle. Le sillon est très souvent rempli par du matériel fin, qui trouve là un abri favorable à son dépôt, le même constat a été fait dans le delta de l'Ebre (GUILLEN \& PALANQUES, 1993). La mobilité de ces rides semble être en relation directe avec l'évolution de la morphologie des plages aériennes.

Les sables plus ou moins fins obéissent à la dynamique marine littorale et infralittorale. Ils alimentent les fonds favorables à leur dépôt mais transitent aussi le long de la côte sous l'effet des courants de dérive littorale approvisionnant ainsi les plages aériennes.

Ces dernières sont soumises à la déflation éolienne qui aboutit à la formation et/ou l'alimentation des dunes vives actuelles. Ces formations sont l'objet de l'essentiel des exploitations intenses et même abusives en granulats qui a abouti à leur destruction totale occasionnant des situations le plus souvent catastrophiques. Afin de cerner ces problèmes des levés de profils de plage perpendiculaires à la côte répartis en quatorze stations ont été réalisés à environ deux mois d'intervalles sur un cycle annuel, de novembre 1983 à octobre 1984. Cette étude a concerné les deux grandes plages : celle de Zemmouri et du Figuier.

Le choix a porté sur quatre stations (V, XI, XIII, XVI) pour illustrer les variations conséquentes à l'engraissement et à l'érosion des plages aériennes (figure 1a).

Ces profils (figure $1 \mathrm{~b}$ ) montrent des largeurs de plage notables en période estivale (levés des mois de mai et de juillet) témoins d'un engraissement et d'une érosion de celles-ci en période hivernale (levés des mois d’octobre et de novembre). Ce schéma classique est bouleversé, étant donné que certains profils à une situation particulière s’érodent en été et s’engraisse l'hiver. 


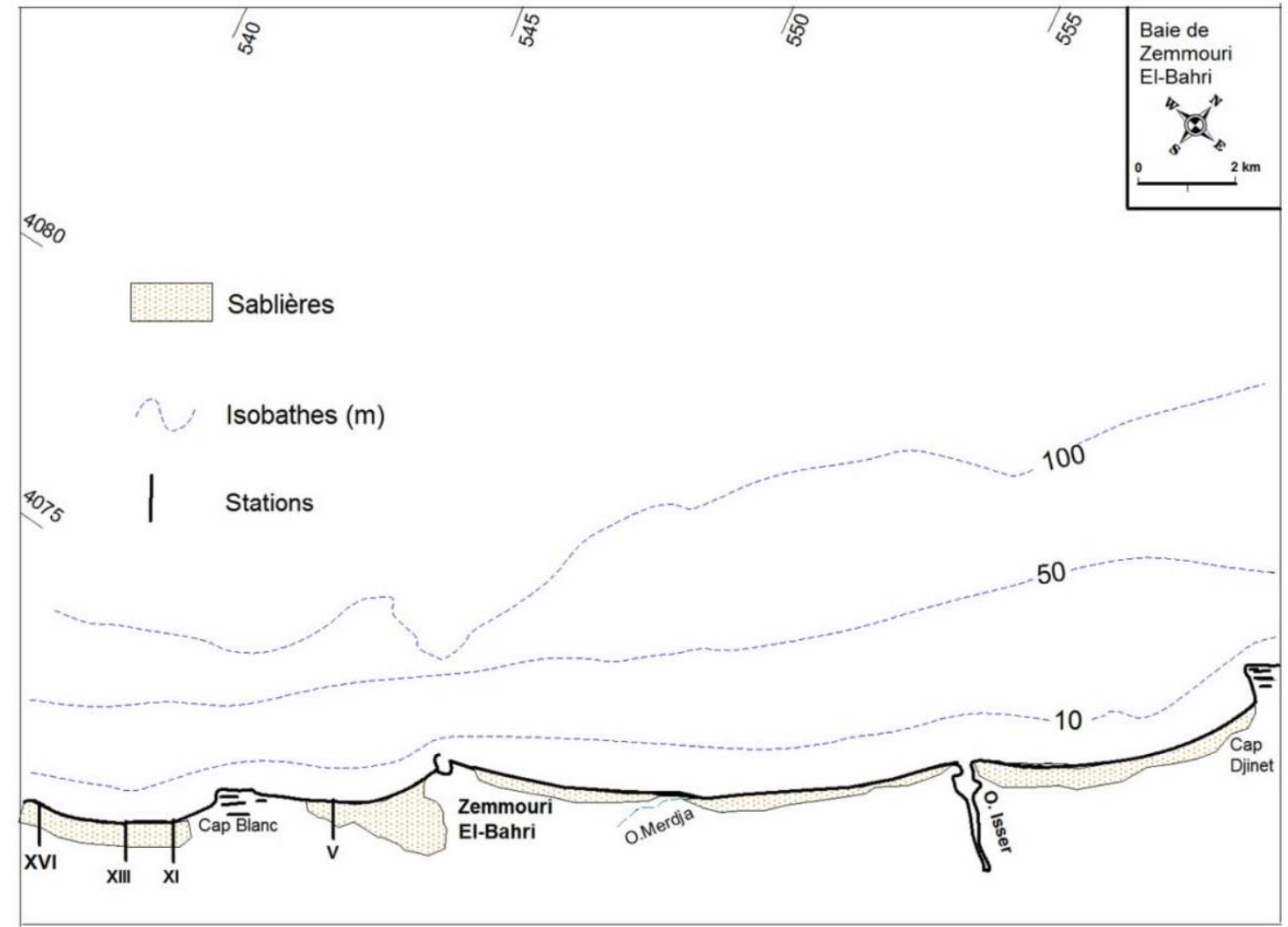

Figure 1a. Localisation des profils de plage et des sablières.
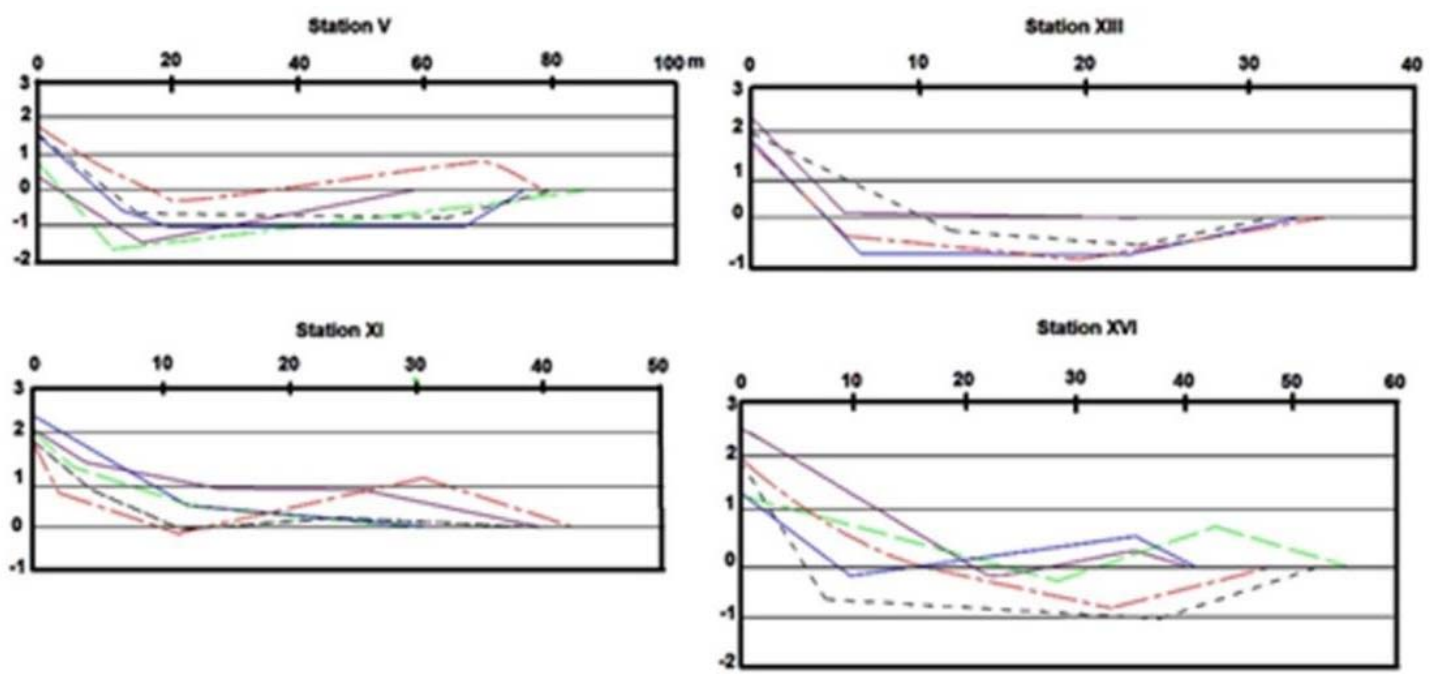

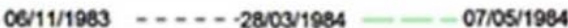

$0307 / 1964$

13/10/1994

Figure 1b. Profils de plage entre Zemmouri et Boumerdes.

Ces derniers se situent dans des zones de sablières dont l'exploitation se traduit par le surcreusement de la plage occasionné par les engins qui agissent en saison estivale. Lors 
Côtes méditerranéennes menacées :

Risques et défis dans le contexte du changement climatique

du déferlement, la mer envahit ces dépressions artificielles et provoque la disparition d'une portion de la plage. Ce phénomène est bien visible aux stations XIII et XVI.

Par ailleurs pour la station V, la comparaison du profil de plage du mois de novembre 83 (période hivernale) et du levé du mois de juillet 84 (la saison estivale) montre une augmentation de la largeur de plage :

- au mois de mars, on assiste à un comblement par les sédiments sur les 40 mètres à partir du début du profil par rapport au mois de novembre, puis disparition totale de la plage ;

- au mois de mai, le profil se creuse d'un mètre au point d'inflexion ;

- au mois de juillet, à la première inflexion du profil, un apport de sédiments sur 1,5 mètre de hauteur, à la deuxième inflexion la plage s'est engraissée d'environ 1 mètre de hauteur et de 20 mètres de largeur par rapport au mois de novembre ;

- au mois d'octobre, il y a un nouveau creusement.

En fait ces creusements qui interviennent après comblement des levés précédents trouvent leur explication dans la surexploitation des sablières.En l'absence de tout contrôle, il nous a été pratiquement impossible de chiffrer cette érosion à la fois naturelle et anthropique. Aussi l'équilibre naturel a été perturbé par l'exploitation en amont de l'estuaire de l'Isser le long du lit de l'oued d'une part, par la rétention des apports suite à la construction de la retenue de Béni-Amrane d'autre part.

\section{Conclusion}

Les variations morphologiques du littoral et la mobilité des rides d'avant côte les plus importants s'observent pendant les périodes de grandes tempêtes.Les rides d'avant côte se déplacent vers la côte, la mer envahit la plage, qui se rétrécit en hiver. Pendant la saison estivale, les rides se déplacent vers le large, de ce fait la plage est plus développée.

L'intervention de l'homme pour extraire le sable peut modifier ce schéma classique, l'érosion aura lieu en été, car les conditions sont favorables à l'exploitation des sablières sans aucune contrainte météorologique.

Le déplacement des rides et l'occupation de leur sommet par du matériel grossier sont une conséquence des courants de retour, responsables de leur édifice et de la sélection de la granulométrie de leurs composants.

\section{Références bibliographiques}

GUILLEN J., PALANQUES A. (1993). Longshore bar and trough systems in a microtidal, Storm-Wave dominated coast: The Ebro Delta (Northwestern Mediterranean). Marine Geology, Vol. 115, pp 239- 252. http://dx.doi.org/10.1016/0025-3227(93)90053-X

PASKOFF R. (2010). Les littoraux: Impact des aménagements sur leur évolution. Armand Colin, Paris, 264 p. 